\title{
Gommunications.
}

\section{Description d'un Rosalia nouveau du Yunnan [Col. Cerambycidae]}

par P.-L. Boppe.

Rosalia Bouvieri, n. sp. - Lutea. Antennarum articulis 3-6 haud spinosis, luteo et nigro annulatis. Pronoto fortiter tuberculato, et in disco maculis duabus nigris, utrinque dentato. Elytrorum humeris duabus maculis nigris; in quarta anteriore parte, maculis quatuor nigris, prima versus suturam, secunda minore versus latus. In disci medio duabus maculis, transverse elongatis et in quarta posteriore parte duabus maculis versus suturam et puncto minore versus latus. - Long. 26 mill.; élytr. 19 mill.; lat. 7 mill.

Fond de la livrée, jaune brunàtre chamois

Antennes à scape noir, finement et nettement chagriné, ce relief diminuant d'intensité vers le sommet, garni en dessous d'une pilosité noire et raide, de mème que le $2^{\text {e }}$ article. Articles 3-6 non épineux, subégaux, garnis de poils raides de la couleur du fond sur la moitié antérieure, noirs sur la partie postérieure, ne laissant à découvert que l'angle interne postérieur qui apparaìt brillant. Les antennes sont ainsi amnelées de jaune et de noir.

Tète noire garnie d'une épaisse pilosité de la couleur du fond, couvrant toute la partie supérieure de la tète sauf les joues, les mandibules et l'épistome et s'étendant, en-dessous, sur la gorge et la ligne médiane du menton.

Prothorax de la couleur du fond, orné en dessus d'une tache trapézoïdale de pilosité noire, en arrière de laquelle s'en trouve une seconde plus petite et de forme arrondie, voisine de la base qui est, en cet endroit, lenticulairement déprimée. Còtés fortement tuberculés, disque présentant latéralement une dent dénudée et brillante.

Mésothorax noir, garni d'une pilosité couleur du fond, de mème que le scutellum.

Saillie prosternale large, celle du mésosternum plus large et fortement pileuse.

Élytres non granuleux à leur base, de la couleur du fond, ornés. dans l'angle huméral d'une tache noire d'environ 1 mill. de diamètre.

Le système de 3 bandes noires habituel dans le genre Rosalia se retrouve ainsi modifié :

La bande du quart antérieur se réduit, sur le milieu de chaque élytre, 
à une tache noire, arrondie, plus petite que la tache humérale, accompagnée sur le côté de l'élytre, et très extérieurement, d'une seconde tache analogue, placée sensiblement en avant de la première.

La bande médiane est oblique par rapport à l'axe, l'ensemble sur les deux élytres formant un angle obtus ouvert vers l'avant. Cette bande, large d'environ 1 mill. au milieu de chaque élytre, s'étend depuis l'épipleure jusqu’à $1 / 2$ mill. du bord sutural, s'é-

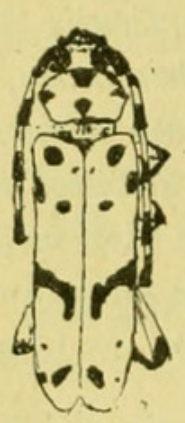

Fig. 1.

Rosalia Bouvieri $\mathrm{B}$ ор pe. largissant à ses extrémités jusqu'à une largeur double. Le bord antérieur de cette bande présentant une forme sinueuse et de direction semicirculaire.

La bande postérieure se compose d'une tache noire allongée s'étendant depuis le milieu de chaque élytre jusqu’à 1/2 mill. du bord sutural, placée obliquement par rapport à l'axe, l'ensemble sur les deux élytres forme un angle ouvert vers l'apex; puis d'une petite tache arrondie se trouvant à la même hauteur que la première mais près du bord épipleural $\left.{ }^{1}\right)$.

Il existe enfin sur chaque élytre un point très petit de pilosité noire en arrière du scutellum et très près du bord sutural, puis un autre également très petit vers le milieu de chaque élytre et à mi-distance des deux bandes médiane et postérieure.

Abdomen couvert sur tous les sternites d'une pilosité couleur du fond. Fémurs fusiformes, noirs, recouverts d'une pilosité couleur du fond s'étendant en anneau interrompu sur les deux faces latérales et le dessus, et laissant à nu les extrémités. Tibias noirs. Tarses non recouverts en dessus de pilosité couleur du fond.

Cette espèce présente en somme, bien que très modifiée, la livrée ordinaire des Rosalia, mais elle est intéressante dans un genre dont toutes les espèces se rapportent à deux types de coloration bien déterminés répondant aux deux genres Rosalia Serville et Eurybatus Thomson. Tandis qu'un fond de coloration bleu cendré se retrouve dans toutes les espèces de Rosalia str. s., il est remplacé d'une manière constante par une teinte vermillon dans celles du genre Eurybatus.

A. Lameere (Mon. du gen. Rosalia in Ann. Soc. Ent. Belg., XXXI, p. 1599) a montré qu'il n'existait entre ces deux genres aucune autre différence essentielle que la coloration et les a réunis en un seul, le genre Rosalia. Notre nouvelle espèce fournit un argument de plus en faveur de sa théorie, répondant à un troisième type différent des deux premiers.

(1) Cette tache très visible sur l'élytre gauche, est presque oblitérée sur l'élytre droit. 
Elle est de plus intéressante par la nature de sa pilosité qui, au lieu de constituer un duvet très serré et très fin, très régulier, est au contraire grossière et bourrue, particulièrement sur les régions inférieures du corps.

Un individu probablement $\$$, incomplet. Collection du Muséum national d'Histoire naturelle.

Yunnan-Tstekou et Se-Tchouen : principauté de Batang Yargong, SOULIÉ 1907.

M. le $\mathrm{P}^{\mathrm{r}}$ Bouvier a bien voulu nous permettre de lui dédier cette jolie espèce dont nous devons la communication à notre collègue II. P. LESNE. Nous leur témoignons à l'un et l'autre notre respectueuse reconnaissance pour l'amabilité avec laquelle ils ont bien voulu nous donner accès au Muséum.

\section{Note sur Gallimoxys Brullei Mulsant [CoL. Cerambycidae]}

\section{par C.-J. Gahan et E. Gounelle.}

Le Callimoxys Brullei Muls., dont le type, qui faisait partie de la collection Chevrolat, se trouve au British Museum, n'est pas synonyme du C. gracilis Brullé comme l'indiquent les catalogues, mème les plus récents. Ainsi que Mulsant le présumait lui-même, c'est une espèce bien distincte qui, non seulement appartient à un genre tout à fait différent, mais encore doit être rayée de la liste des Coléoptères d'Europe.

Sa patrie en effet n'est ni la Grèce ni la Dalmatie, comme l'a écrit Mulsant trompé par une étiquette erronée, mais l'Amérique du Sud où son aire de dispersion assez vaste comprend les régions méridionales du Brésil intérieur, le Paraguay, le nord de la République Argentine et même une partie de la Bolivie (un exemplaire originaire de cette contrée, nommé Cosmosoma aereipenne (Chevrol. in litt.), fait partie des collections du British Museum).

Le $C$. Brullei a été décrit de nouveau par Burmeister (Stett. Zeit., [1865], p. 171) sous le nom de Cosmosoma nodicolle sur un individu provenant du Parana. C'est bien en effet un Cosmosoma; mais l'auteur ayant placé dans sa diagnose la touffe de poils qui orne les antennes sur le sixième article, alors qu'en réalité elle se trouve sur le cin- 


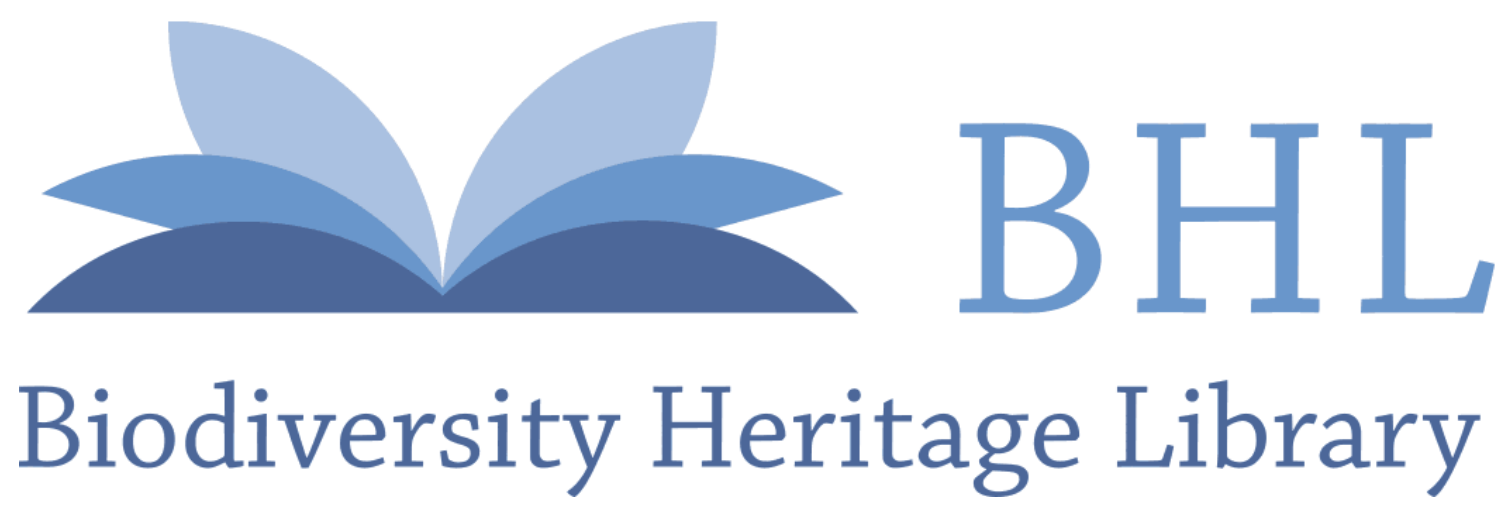

1910. "Description d'un Rosalia nouveau du Yunnan." Bulletin de la Société entomologique de France 1910, 235-237. https://doi.org/10.5962/bhl.part.4678.

View This Item Online: https://www.biodiversitylibrary.org/item/38239

DOI: https://doi.org/10.5962/bhl.part.4678

Permalink: https://www.biodiversitylibrary.org/partpdf/4678

\section{Holding Institution}

Smithsonian Libraries

\section{Sponsored by}

Smithsonian

\section{Copyright \& Reuse}

Copyright Status: NOT_IN_COPYRIGHT

This document was created from content at the Biodiversity Heritage Library, the world's largest open access digital library for biodiversity literature and archives. Visit BHL at https://www.biodiversitylibrary.org. 